\title{
Programas guía de actividades desde la resolución de problemas: una estrategia contextual de intervención didáctica en electroforesis
}

\author{
PGA since problem solving: a contextual strategy for didactic intervention to teach \\ electrophoresis
}

\author{
Laura Milena Cala Cristancho', Leonardo Mariño Repizo ${ }^{1}$ y Jaime Augusto Casas ${ }^{2}$
}

1 Estudiantes de licenciatura en Química. Universidad Pedagógica Nacional; Profesor del Departamento de Química Universidad Pedagógica Nacional. Bogotá, Colombia. b laito cal@hotmail.com, leodc 9@hotmail.com, jcasas@pedagogica.edu.co

\section{Resumen}

Este trabajo se realizó durante el primer período del 2009, con estudiantes de grado décimo del énfasis en Biotecnología del Instituto Pedagógico Nacional (IPN). Se desarrolló un Programa Guía de Actividades (PGA) basado en la resolución de problemas en contexto con el fin de lograr una transformación en la metodología y las representaciones de los estudiantes sobre el concepto de proteína que fue aplicado al estudio de las proteínas de la leche y bebidas lácteas a través de la electroforesis. Se obtuvieron resultados favorables en cuanto al desarrollo de actividades que permitieron la construcción del concepto de proteína por parte de los estudiantes en la resolución de problemas desde una temática transversal.

\section{Palabras clave}

Resolución de problemas, programa guía de actividades, proteínas, electroforesis, leche.

\section{Abstract}

This study was performed during the first period of this year, with tenth grade students with emphasis on Biotechnology that belonged to the Instituto Pedagógico Nacional (IPN). It was developed a Program Activity Guide (PAG) based on solving problems in context to achieve a transformation in the methodology and in the students' performances about the protein concept applied to the study of milk proteins and dairy beverages by electrophoresis. We obtained favorable results in terms of development activities that led to the construction of the protein concept from the students to problem solving in a cross-thematic.

\section{Keywords}

Problem solving, Program Activity Guide, proteins, electrophoresis, milk.

\section{Introducción}

El uso de temáticas transversales es uno de los caminos más pertinentes y adecuados para desarrollar actividades que involucran la resolución de problemas. Una de estas son los alimentos, espeć́ficamente la leche, que es un alimento básico dentro de los requerimientos nutricionales por su contenido de proteínas, así como otras biomoléculas indispensables para el desarrollo humano. La electroforesis como técnica de análisis se constituye en un "pretexto" para la enseñanza y el aprendizaje de conceptos involucrados través de la resolución de problemas, y es desde este enfoque es conveniente aplicar los PGA como herramientas para la transformación metodológica de las prácticas de enseñanza. 
En esta investigación se plantea el siguiente problema: ¿Qué aportes y transformaciones se pueden dar en el cambio metodológico y en las representaciones de los estudiantes de grado 10-04 del Instituto Pedagógico Nacional (IPN) respecto al concepto de proteína, al aplicar un PGA desde la resolución de problemas empleando la electroforesis para el estudio de las proteínas presentes en la leche?

Para el desarrollo del planteamiento del problema es necesario mencionar algunos referentes conceptuales, tales como:

\section{¿Qué es un problema?}

Como menciona Valle Coronel y Curotto (2008), la enseñanza de los conceptos en química y la metodología empleada por parte de los profesores y estudiantes para aprender y enseñar ciencias se ha centrado y ha predominado en la operatoria algorítmica por sobre todo aspecto, lo que en muchos casos ha limitado al estudiante a la mera repetición de pasos que han impedido la verdadera comprensión de los fenómenos químicos. Por lo tanto, se suele confundir "ejercicios" (de lápiz y papel) con problemas (duros o verdaderos). Esto se debe a que un problema se puede definir una situación que tiene cierto grado de incertidumbre y que tiende hacia la búsqueda de una posible solución, y que además no tiene una vía definida de resolución, sino que presenta diversas posibilidades (Perales Palacios, 1993). Por otro lado, los ejercicios, de forma muy marcada y general, presentan el uso de algoritmos y mecanismos predichos o preestablecidos. Por lo anterior, un problema se define y se diferencia según la vía de resolución. Además, cuando estos problemas son propuestos desde el ámbito escolar, es muy poco relevante el resultado, sino que priman las condusiones o el planteamiento de nuevos problemas (Garća, 2003).

¿Qué es la resolución de problemas?

La resolución de problemas se ha constituido como una línea de investigación que promueve el cambio metodológico, la restructuración de conceptos, así como los procedimientos y actitudes del aprendizaje. La resolución de problemas se constituye en una forma de construcción de conocimiento y desarrollo de habilidades de pensamiento (García, 2000) a través de la realización de procedimientos y métodos basados en la activación de la creatividad, la proposición, la participación, etc.

¿Qué es un Programa Guía de Actividades (PGA) y cómo se relacionan con la resolución de problemas?

Los PGA, son propuestas de desarrollo de unidades didácticas, que se basan en la necesidad de favorecer la construcción de los conocimientos por parte de los alumnos, para lograr que se familiaricen con algunas caractenísticas del trabajo científico. Las unidades didácticas que constituyen un PGA deben estar estratégicamente diseñadas bajo algunos criterios, tales como:

Definición de objetivos: deben hacer referencia a la finalidad de la enseñanza.

$>$ Definición de contenidos o temáticas: deben tener relación entre la ciencia escolar y la de los científicos, deben tener significatividad (por ejemplo las temáticas transversales) y deben posibilitar la comprensión de fenómenos y la relación con el entorno.

$>$ Selección y secuenciación de actividades: un PGA está estructurado con actividades que permitan plantear situaciones que favorezcan la evolución de las ideas o representaciones de los estudiantes. Generalmente se estructuran con actividades de iniciación, actividades de desarrollo o evolución, actividades de síntesis o acabado y actividades de aplicación a nuevos contextos.

$>$ Selección y secuenciación de las actividades de evaluación: la evaluación, autoevaluación y la co-evaluación hace parte de la construcción del conocimiento. 
De esta manera los PGA permiten al estudiante enfrentarse con el planteamiento de hipótesis, identificación de variables y la resolución de problemas, que favorecen la construcción del conocimiento de una manera organizada y estructurada.

Diseño metodológico del PGA fases:

El programa guía de actividades tuvo una duración de dos meses y se estructuró en cuatro

> Fase de iniciación: se aplicó un instrumento en el que se estudiaron las representaciones de los estudiantes respecto al concepto de biomoléaulas, proteínas y otros relacionados, empleando una prueba semántica, en donde se induía la construcción de un texto corto sobre los conceptos requeridos para la temática; además se induyeron preguntas para evidenciar aspectos de carácter metodológico y actitudinal, con el objeto de indagar los intereses de los estudiantes acerca de la temática transversal.

> Fase de contextualización y planteamiento del problema: se realizó la presentación de un video mostrando los avances realizados en la medicina a partir del estudio de las proteínas (proteómica), con la intención de contextualizar la temática presentando una aplicación real. Consecutivamente se generó una plenaria donde se recogieron las afirmaciones por parte de los estudiantes. Además, es importante anotar que en cada sesión y actividad se evaluaron los componentes conceptuales, metodológicos y actitudinales. Las sesiones de la fase de contextualización incluyeron las siguientes actividades, que favorecieron la delimitación del problema a trabajar con los estudiantes:

D Lectura "¿Qué sabes de la leche?".

$>\quad$ Prueba cualitativa para la identificación de los componentes principales de la leche "Reconociendo los componentes de la leche"

> Video titulado "Las proteínas: los robots de la vida"

> Noticia "Posibles hallazgos de adulteración en bebidas lácteas escolares".

> Planteamiento de la hipótesis: ¿es posible determinar mediante un estudio electroforético de proteínas, la adulteración de bebidas lácteas escolares conociendo el contenido de proteínas como la lactoalbuminas y lactoglobulinas?

> Fase de desarrollo: Estas actividades permitieron abordar y buscar una posible solución al problema planteado por los estudiantes a través de las siguientes actividades:

> Taller expositivo sobre los fundamentos y la importancia de la electroforesis SDS-PAGE, mostrando el desarrollo histórico de dicha técnica.

$>$ Práctica experimental: análisis por electroforesis en SDS-PAGE para identificar las proteínas presentes en las bebidas lácteas.

$>$ Socialización de los resultados obtenidos en la resolución del problema a través de un caso simulado.

D Fase de acabado: Esta fase contempla la evaluación la efectividad del PGA en lo referente a los cambios metodológicos y a nivel de las representaciones sobre el grupo objetivo, que se evaluó también con el caso simulado y el instrumento de la fase inicial.

\section{Resultados}

En la primera fase se determinó que los estudiantes establecían muy pocas relaciones para los conceptos de biomoléculas y otros conceptos relacionados, y también que sus representaciones se encontraban distanciadas de los conceptos establecidos por la comunidad científica, espećficamente en el tópico de proteínas. También se evidenció un alto grado de interés por el estudio de temáticas nuevas para ellos, como en el caso de la electroforesis. 
Durante la contextualización, los estudiantes mostraron también un gran interés al realizar preguntas sobre la temática y algunos temas relacionados, como en el caso de los alimentos, las enfermedades, las nuevas medicinas, etc.

Al plantear el problema, los estudiantes se organizaron por grupos de trabajo, en los que cada uno estableció una hipótesis, en la socialización se realizó una lluvia de ideas de cada grupo, y finalmente el problema planteado en consenso fue: ¿es posible determinar mediante un estudio electroforético de proteínas, la adulteración de bebidas lácteas escolares conociendo el contenido de lactoalbuminas y lactoglobulinas como proteínas?

Durante los trabajos prácticos de laboratorio (TPL), los estudiantes se organizaron metodológicamente para desarrollar cada uno de los procedimientos planteados y así dar solución a la problemática. Los estudiantes determinaron que dos muestras de bebidas lácteas, correspondientes a X3 y X5 contenían una mayor cantidad de proteínas en comparación con las demás, en donde su proporción fue normal. De esta manera, los estudiantes comprobaron que es posible determinar a través de la electroforesis, el exceso de suero lácteo como adulterante de las bebidas lácteas.

En el caso simulado, cada uno de los grupos defendió su postura, y establecieron todas las implicaciones que tiene el estudio de las proténas en bebidas lácteas a través de la electroforesis, como las implicaciones industriales, comerciales, nutricionales, económicas, en la salud y finalmente las cient́ficas.

Además de lo anterior, en los resultados de la prueba final, los estudiantes establecieron de una manera más estructurada el concepto de biomoléculas, proteínas, y sus conceptos relacionados; también los estudiantes mostraron un cambio en sus representaciones. Además de esto, les generaron actividades favorables hacia las metodologías trabajadas. Este tipo de investigación permitió que los estudiantes plantearan sus propios proyectos de investigación para ser presentados en EXPOCIENCIA, la feria de la ciencia a nivel académico en nuestro país.

\section{Conclusiones}

El uso del programa guía de actividades basado en la resolución de problemas permitió organizar y a la vez evaluar el aprendizaje de los estudiantes, proporcionando un componente importante en la investigación docente. Igualmente, favoreció el aprendizaje significativo de los conceptos involucrados en electroforesis mediante una construcción de conocimientos fundados en criterios esenciales de la metodología cientúfica. Finalmente, el estudiante desarrolló habilidades que le podrán a futuro servir de utilidad en cualquier actividad de su vida diaria. El estudio aportó de manera significativa al avance en la promoción de nuevas perspectivas metodológicas y a la transformación de sus representaciones sobre conceptos de índole científica frente a temáticas transversales; como valor agregado se encontró que se aprendieron conceptos nuevos para ellos, como lo concerniente al proceso de electroforesis, que es una temática que se pudiera considerar en las aulas universitarias.

Recomendaciones: La elaboración de las actividades a trabajar dentro del PGA debe enfocarse en la construcción metodológica, que acerque el trabajo de la comunidad cient́fica al aula, con la intención de proporcionar variedad de mecanismos para resolver problemas cotidianos.

Agradecimientos: Agradecemos especialmente a las directivas, profesores y estudiantes del Instituto Pedagógico Nacional, así como al Departamento de Química de la Universidad Pedagógica Nacional por su inmensa colaboración para el desarrollo del presente proyecto. 


\section{Bibliografía}

García, J. J. (2003). Didácticas de las ciencias: Resolución de problemas de desarrollo de la creatividad Bogotá, Colombia: Didácticas Magisterio. pp. 34 y 56.

García, J. J. (2000). La solución de situaciones problemáticas: una estrategia didáctica para la enseñanza de la química. Enseñanza de las ciencias. 18 (1), 113-129.

Perales, F.J. (1993). La resolución de problemas: una revisión estructurada. Enseñanza de las ciencias. 11 (2), 170-178.

Valle, M.V y M.M Curotto (2008). La resolución de problemas como estrategia de enseñanza y aprendizaje Revista Electrónica de Enseñanza de las Ciencias 7, 2: 463-479. En http: //www.saum uvigo.es/reec 